\section{Revue d'ethnoécologie}

$20 \mid 2021$

Un siècle d'ethno-machins : le centenaire de la Revue de Botanique Appliquée

\title{
Orígenes, evolución y retos de la etnobiología boliviana
}

Origines, évolution et défis de l'ethnobiologie bolivienne

Origins, evolution and challenges of Bolivian ethnobiology

Victoria Reyes-García, Mónica Moraes R., Álvaro Fernández-Llamazares y Narel Y. Paniagua-Zambrana

\section{OpenEdition}

\section{Journals}

Edición electrónica

URL: https://journals.openedition.org/ethnoecologie/8164

DOI: 10.4000/ethnoecologie.8164

ISSN: 2267-2419

Editor

Laboratoire Éco-anthropologie

\section{Referencia electrónica}

Victoria Reyes-García, Mónica Moraes R., Álvaro Fernández-Llamazares et Narel Y. Paniagua-

Zambrana, "Orígenes, evolución y retos de la etnobiología boliviana », Revue d'ethnoécologie [En ligne], 20 | 2021, mis en ligne le 31 décembre 2021, consulté le 06 mars 2022. URL : http://

journals.openedition.org/ethnoecologie/8164; DOI : https://doi.org/10.4000/ethnoecologie.8164

Este documento fue generado automáticamente el 6 marzo 2022.

\section{C)}

Revue d'ethnoécologie est mis à disposition selon les termes de la licence Creative Commons Attribution - Pas d'Utilisation Commerciale - Pas de Modification 4.0 International. 


\title{
Orígenes, evolución y retos de la etnobiología boliviana
}

\author{
Origines, évolution et défis de l'ethnobiologie bolivienne \\ Origins, evolution and challenges of Bolivian ethnobiology
}

Victoria Reyes-García, Mónica Moraes R., Álvaro Fernández-Llamazares y Narel Y. Paniagua-Zambrana

\section{Introducción}

1 La etnobiología estudia las relaciones que las sociedades entablan con la naturaleza, analizando conocimientos, usos y percepciones (Toledo et al. 2002, Sillitoe 2006, ReyesGarcía \& Martí-Sanz 2007). En su conjunto, la etnobiología aporta una visión dinámica de estas relaciones basada en el entendimiento de procesos históricos y enfatizando que las interacciones entre aspectos sociales y naturales pueden ser bidireccionales (Sillitoe 2006 Hunn 2007).

2 Aunque el término etnobiología no fue acuñado hasta el siglo $\mathrm{XX}$, dos disciplinas académicas mostraron antes interés por las relaciones que las diferentes sociedades establecen con la naturaleza: la biología y la antropología. Por un lado, desde el siglo $\mathrm{XV}$, los naturalistas se interesaron por el conocimiento local elaborando listas de animales y plantas conocidos por una sociedad, así como sus usos. Este estudio del conocimiento local informó algunos aspectos clave del desarrollo de disciplinas como la biología, incluyendo la clasificación taxonómica de Linneo (Ellen 2004). Por otro lado, desde sus orígenes, la antropología también mostró interés en las relaciones que diferentes grupos sociales mantienen con la naturaleza. Este interés dio origen a distintas corrientes en el seno de la antropología, y en particular una subdisciplina enmarcada dentro de la antropología cognitiva conocida como etnoecología (Milton 2010). En sus inicios en la década de los años 1950, la etnoecología centró en documentar sistemas de clasificación de diferentes elementos naturales, como las plantas o el suelo (Berlin et al. 1966, Hunn 1982). Dicho interés venía motivado por la búsqueda de principios universales en los procesos cognitivos de clasificación, aunque 
pronto evolucionó hacia el estudio general de los sistemas de conocimiento mediante los que las diferentes sociedades gestionan el entorno natural (Conklin 1954, Atran 1985, Posey 1985).

3 Los estudios de carácter propiamente etnobiológico, es decir concediendo el mismo rigor a aspectos sociales y biológicos y enfatizando la importancia de las interacciones entre éstos, fueron relativamente marginales hasta la década de 1990, cuando el conocimiento ecológico local ${ }^{1}$, considerado hasta el momento como rudimentario y superfluo, empezó a permear documentos políticos como Nuestro Futuro Común (1987) o la Convención de Diversidad Biológica (1992). Esta coyuntura activó el reconocimiento de la importancia del conocimiento ecológico local por parte de científicos, activistas, tomadores de decisiones y el público en general. Desde la década de los años 1990, numerosas investigaciones han enfatizado el valor del conocimiento ecológico local presentándolo como resultado y estrategia de la adaptación humana al medio ambiente (Berkes et al. 2000, Toledo et al. 2002, Reyes-García et al. 2016). En este contexto, también nace la idea de que el conocimiento ecológico local puede ser de utilidad para la elaboración de programas de conservación y restauración ecológica y para la gestión sostenible de los recursos naturales (Huntington 2000, Pitcher 2001).

Este cambio de paradigma en la valoración del conocimiento ecológico local coincidió con una transformación en la orientación de los programas de conservación y desarrollo, que empezaron a considerar la importancia de involucrar a las poblaciones beneficiarias en el diseño y la gestión de dichos proyectos. En este nuevo contexto, ciertos elementos del conocimiento ecológico local, como por ejemplo el manejo de los recursos naturales, se mostraron de gran utilidad práctica para dichos proyectos (Sillitoe 2006). Siguiendo esta línea, en las últimas dos décadas, la etnobiología ha enfatizado su dimensión aplicada, contribuyendo a discusiones sobre la importancia de la co-gestión de los recursos naturales o el papel de los pueblos indígenas y las comunidades locales en la gobernanza ambiental (Boillat et al. 2013, Armstrong \& McAlvay 2019, Reyes-García et al. 2022). Recientemente, el conocimiento detentado por pueblos indígenas y comunidades locales ha tomado mayor relevancia al empezar a ser incluido, no solo en proyectos de carácter local, sino también en políticas globales. Por ejemplo, la reciente evaluación llevada a cabo por la Plataforma Intergubernamental Científico-Normativa sobre Diversidad Biológica y Servicios de los Ecosistemas (IPBES) afirma que "los sistemas de conocimientos indígenas y locales tienen enfoques locales, pero se manifiestan a nivel regional $y$, por ende, atañen a todo el mundo" (IPBES 2019). En este contexto, va ganando fuerza la idea de que el conocimiento, las prácticas y los valores que forman el conocimiento ecológico local pueden contribuir a dar respuestas a la crisis de biodiversidad en la que nos encontramos (Reyes-García et al. 2022).

5 Más allá de estas tendencias generales, entender el desarrollo de la investigación etnobiológica requiere profundizar en su desarrollo en contextos específicos, pues es dicha diversidad la que conforma el patrón global. Con el objetivo de contribuir a ese fin, en este trabajo analizamos los orígenes, evolución, y retos de la etnobiología boliviana. Bolivia ofrece un excelente caso de estudio para este fin por dos razones. En primer lugar, con 36 naciones originarias y 32 regiones ecológicas distintas, es un país de importante diversidad biológica y cultural (Ibisch \& Mérida 2003, Romero-Muñoz et al. 2019). Durante años, este rico patrimonio biocultural ha captado el interés de científicos bolivianos y extranjeros (p. ej., Orlove \& Godoy 1986, Toledo 1996, Scalise Sugiyama et al. 2020), por lo que el estudio del contexto boliviano nos permite ver las 
interacciones entre los contextos nacional e internacional. En segundo lugar, Bolivia se encuentra actualmente en un proceso sociopolítico de revalorización de los sistemas de conocimiento local y de defensa de la identidad y la gobernanza indígena (Laing 2015, Turner 2019). La firme apuesta por los programas de educación y salud interculturales, así como la mayor participación indígena en la gestión ambiental, han promovido el interés en la etnobiología como herramienta para visibilizar, reconocer y movilizar la gran amalgama de conocimientos de los pueblos indígenas bolivianos (Mathez-Stiefel et al. $2012 \mathrm{a}, \mathrm{b}$, Moraes et al. 2016). Sin embargo, este proceso se enfrenta a varios retos que pueden afectar el éxito de dichos programas, y para los que la etnobiología debe estar preparada.

6 Para cumplir los objetivos de este trabajo, en la siguiente sección analizamos los inicios de la etnobiología en Bolivia desde sus orígenes biológicos y antropológicos. Esta sección no aspira a ser exhaustiva, sino a trazar los mayores rasgos de la historiografía del desarrollo de la disciplina. En la tercera sección analizamos tres estudios etnobiológicos desarrollados en la Amazonía boliviana. Nos centramos en la región amazónica, ya que la etnobiología andina cuenta con una mayor base documental y ha recibido generalmente mayor atención académica. Los dos primeros estudios muestran la convergencia de un estudio de carácter biológico y uno de carácter antropológico y el tercero ejemplifica el enfoque participativo. La última sección analiza los retos de la disciplina en Bolivia, en relación tanto al desarrollo político del país como a la evolución de la disciplina a nivel internacional.

\section{Orígenes de la etnobiología en Bolivia}

\section{Precursores biológicos de la etnobiología boliviana}

7 Hasta la década de 1990, la mayoría de los estudios biológicos que incluían información proporcionada por pueblos indígenas y comunidades locales de Bolivia se centraba en analizar listados de especies y usos, ocasionalmente incluyendo detalles puntuales sobre el manejo y procesamiento de las especies (p. ej., Boom 1987, Girault 1987, Vidaurre et al. 2006). En estos estudios, la investigación y relevamientos en campo se planificaban priorizando la documentación de las características y patrones de la biodiversidad en diferentes paisajes de la geografía boliviana y la información se recopilaba como parte del registro sistematizado en las notas de campo. Este es el caso, por ejemplo, de las crónicas de viaje del naturalista francés Alcides d'Orbigny, quien entre 1830 y 1833 documentó los nombres vernaculares y el uso que varios pueblos indígenas de Bolivia daban a 40 especies de palmeras (Martius 1843).

Los primeros trabajos realizados en Bolivia que tuvieron el conocimiento ecológico local como principal objeto de estudio aparecen hacia finales de la década de los 1980 y fueron relevamientos etnobotánicos. Así, en un trabajo pionero sobre los curanderos kallawaya, Girault (1987) documentó 170 géneros y ca. 900 especies con uso medicinal. Otros ejemplos destacados incluyen los estudios de De Lucca \& Zalles (1992) y LozaBalsa (1995) entre los aymara y quechua del altiplano y los estudios sobre la medicina tradicional de los valles interandinos (p. ej., Alba 1993, Pestalozzi \& Torrez 1998). De forma similar, Hensen (1992) documentó 112 especies de uso medicinal y 25 plantas silvestres alimenticias entre campesinos de la comunidad Chorojo (Cochabamba). En las tierras bajas, destaca el trabajo de Boom (1987) entre los chácobo, quien documentó 305 
plantas útiles. En bosques montanos de yungas, las primeras tesis universitarias etnobotánicas investigan las plantas utilizadas por los mosetenes (Hinojosa 1991) y los muchanes (Vargas 1996). En la misma línea de trabajo, DeWalt y colaboradores (1999) evaluaron las plantas útiles del pueblo tacana y encontraron que el $64 \%$ de las 115 especies documentadas tenía más de un uso; mientras que Bourdy y colaboradores (2000) relevaron aplicaciones medicinales de 148 plantas. Bourdy (2002) también investigó las plantas útiles de los guaraní del Chaco y Vandebroek y colaboradores (2004) estudiaron las plantas medicinales de los yuracaré, que más tarde fueron comparadas con la etnofarmacología de los Andes y la Amazonía (Thomas 2009, Thomas et al. 2009a). Recientemente, también se han realizado estudios etnobotánicos en mercados urbanos especializados en la venta de plantas medicinales (p. ej., Macía et al. 2005).

Además de documentar los usos de las plantas silvestres, los precursores biológicos de la etnobiología boliviana también han contribuido a documentar procesos de domesticación realizados por pueblos indígenas y comunidades locales en sus sistemas de producción, incluyendo la selección y mejoramiento de cultivos para alimentos, aceites, frutos, fibras y otros usos (p. ej., Cárdenas 1969, Gandarillas 1979, Rea 1992). Esta línea de trabajo también ha contribuido a identificar centros de origen y recursos fitogenéticos y a documentar la diversidad vegetal agrícola manejada por los pueblos indígenas y comunidades locales (Moraes et al. 1996), así como a documentar el conocimiento local de suelos (Zimmerer 1994). Finalmente, también desde los intereses biológicos, se han promovido experiencias comunitarias para manejo de fauna silvestre que han contribuido a la sostenibilidad de la caza de animales nativos (Townsend 2000, Townsend \& Rumiz 2004).

\section{Precursores antropológicos de la etnobiología boliviana}

En Bolivia, el interés antropológico por la relación entre los grupos humanos y su medio ambiente inicialmente vino de autores extranjeros interesados en describir la diversidad cultural mediante trabajos puramente etnográficos. Este es el caso de los relatos del etnógrafo sueco-finlandés Nordenskjöld, quien recorrió Bolivia en los años 1920 dejando un amplio legado de observaciones lingüísticas y culturales, incluyendo muchos usos de animales y plantas (Nordenskjöld 1924). Durante la década de los años 1970, el interés de autores extranjeros por la diversidad cultural boliviana toma un carácter más analítico que se plasma en trabajos que describen la estructura social de diferentes grupos indígenas (p. ej., Albó 1972, 1979), su historia (p. ej., Barnadas 1976), o la integración ecológica (Harris 1978). En ese periodo, destaca también el análisis de la verticalidad andina del boliviano Concardo Morales (1978). Estos trabajos suponen una diferencia con el enfoque etnográfico ya que incluyen análisis orientados a entender cómo la organización social se relaciona con la gestión de los recursos naturales, constituyendo por tanto importantes precursores antropológicos de la etnobiología boliviana (Salomon 1985). Cabe destacar que estos trabajos quedan lejos de las tendencias internacionales del momento, generalmente centradas en entender sistemas locales de clasificación (p. ej., Berlin et al. 1973, Berlin 1976).

Con la excepción del trabajo de Concardo Morales, el interés en las relaciones entre los grupos humanos y su ambiente es más tardío en la antropología que en la biología boliviana. Esta situación está probablemente relacionada con el desarrollo de la 
disciplina en el país ya que, pese a la alta diversidad cultural, la antropología no se institucionalizó en Bolivia hasta la década de 1980 (Barragán 2009). Es más, en sus inicios, la antropología boliviana estuvo dominada por debates relacionados con el concepto de "clase", una tendencia que probablemente refleja las preocupaciones de las élites intelectuales bolivianas que acceden a los estudios universitarios y que intentan desmarcarse de los pueblos indígenas y comunidades locales, a menudo considerados social y culturalmente obsoletos (ver Barragán 2009).

12 Así, no es hasta la década de los 1990 cuando la cuestión indígena empezó a dominar en la antropología boliviana en un proceso dirigido por investigadores e instituciones bolivianas como el Centro para la Investigación y Promoción del Campesino (CIPCA) y el Centro para el Estudio de la Realidad Económica y Social (CERES) (Larson 2019). Cabe destacar que los estudios en esta línea estaban abiertamente politizados, reclamando la esencia indígena de Bolivia y respaldando la lucha de los pueblos indígenas en defensa de sus derechos (Rivera Cusicanqui 1999, Larson 2019). Por ejemplo, los trabajos antropológicos de la década de 1980 documentando la ocupación territorial indígena durante los siglos XV y XVI (p. ej., Bouysse-Cassagne 1980, Saignes 1986, Wachtel 1990) son utilizados para apoyar demandas territoriales. A mediados de la década de los noventa, el reconocimiento político del término "Pueblo Indígena" en Bolivia contribuyó a alentar las demandas por el reconocimiento de las lenguas y culturas locales, así como el derecho al territorio (Larson 2019). Este proceso fue acompañado por un aumento de estudios antropológicos sobre la política agraria, los derechos de los pueblos indígenas, el desarrollo local, el empoderamiento, o la discriminación de las mujeres indígenas (Larson 2019). Sin embargo, y pese a que muchos de esos trabajos abordan de forma indirecta las relaciones de los pueblos indígenas con la naturaleza, pocos tienen un carácter explícitamente etnobiológico. En Bolivia, los trabajos propiamente etnobiológicos liderados desde la antropología no se popularizan hasta los años 2000, a menudo liderados por equipos internacionales (p. ej., Godoy et al. 2005, Reyes-García et al. 2003, 2006).

13 Es importante subrayar que, pese a que la antropología cultural boliviana tuvo un acercamiento tardío a la etnobiología, existen acercamientos desde otras disciplinas de las ciencias sociales como la arqueología (Langlie et al. 2011, Prestes-Carneiro \& Béarez 2017), la ecología histórica (Denevan 1966, Erickson 2011), o la geografía (Sarmiento 2010). Por ejemplo, entre los estudios clásicos en los que confluyen la arqueología y la ecología histórica destaca el trabajo en los Llanos de Moxos (Denevan 1966, Erickson 2011). Al analizar el hábitat pre-colombino de los humedales, bosques y sabanas de esta región amazónica, estos trabajos muestran cómo la arqueología puede proporcionar una visión de largo plazo sobre el cambio ambiental, contribuyendo a la gestión sostenible de los recursos naturales (p. ej., Mann 2008, Lombardo et al. 2020).

\section{La formalización de la etnobiología como disciplina científica en Bolivia}

Fruto de la creciente confluencia de intereses en el estudio de las interrelaciones entre las sociedades y la naturaleza, en 2008 se formalizó la etnobiología en Bolivia con la creación de la Sociedad Boliviana de Etnobiología-AGRUCO en Cochabamba. Posteriormente, se han organizado eventos nacionales e internacionales que han contribuido a la consolidación de la disciplina. A nivel nacional, el Instituto de Ecología 
y el Herbario Nacional de Bolivia de la Universidad Mayor de San Andrés (UMSA), con el apoyo del Institut de Ciència i Tecnologia Ambientals de la Universitat Autònoma de Barcelona (ICTA-UAB), han organizado tres jornadas $(2015,2017$, y 2019) para el intercambio de avances científicos en etnobiología en Bolivia (https:// etnobiologialpbict.wixsite.com/jornadas). Estas jornadas han contado con la participación de investigadores y estudiantes de biología, antropología, ingeniería ambiental, y agronomía, así como de representantes de pueblos indígenas y organizaciones no-gubernamentales y han emitido manifiestos con el posicionamiento de los participantes (Moraes et al. 2016). Además, desde 2017, la carrera de biología de la UMSA incluye la asignatura de etnoecología en la malla curricular para la maestría en biología.

A nivel internacional, Bolivia ha acogido en dos ocasiones el Congreso Latinoamericano de Etnobiología (CLAE), en 2012 en La Paz, donde el Instituto de Ecología de la UMSA organizó el III CLAE, y en 2019 en Sucre, donde se celebró el VI CLAE con el respaldo de la organización no gubernamental Comunidad Pluricultural Andino-Amazónica para la Sustentabilidad (COMPAS). Se puede considerar, por tanto, que la disciplina avanza hacia su consolidación en Bolivia.

\section{La evolución de la etnobilogía boliviana: Tres estudios de caso de la Amazonía boliviana}

En esta sección analizamos en profundidad la evolución de la etnobiología en Bolivia mediante tres estudios desarrollados en la Amazonia boliviana. Siguiendo la línea argumental de la sección precedente, los dos primeros estudios muestran respectivamente la evolución desde la biología y desde la antropología hacia la etnobiología, mientras que el tercero describe el enfoque participativo en la investigación etnobiológica.

\section{De la biología a la etnobiología: los tacanas y las palmeras de Tumupasa}

Los tacanas son un pueblo indígena lingüísticamente perteneciente al grupo panotacana (Lehm 2016). Actualmente viven en zonas de Pando, Beni y norte de La Paz, donde la mayoría de la población se concentra en el Territorio Comunitario de Origen Tacana 1 (Tejeiro 2010). El grupo es conocido por su capacidad de liderazgo, documentada desde el siglo XVI con trabajos que sugieren que los jefes tacanas disfrutaban de importantes cuotas de poder y gobernaban extensos territorios y poblaciones relativamente numerosas (Brohan \& Herrera 2008, Lehm 2016). Aunque los tacanas fueron parte de reducciones, p. ej. Tumupasa, no abandonaron completamente su cultura, por lo que su organización sociopolítica se ha mantenido como centro de reproducción cultural hasta ahora (Lehm 2016). Como otros pueblos indígenas, el pueblo tacana ha manejado su entorno natural mediante el conocimiento aplicado al desarrollo de sistemas de uso de la tierra, tipos de cacería, y procedimientos culturales basados en creencias, tradiciones y control social (Hahn \& Hissink 2000). Sin embargo, procesos impuestos en su territorio, como la creación de áreas protegidas o la extracción de recursos, han resultado en la modificación y adaptación de estas prácticas (Díez Astete 2011). Esto, a su vez, ha llevado a la aparición de nuevas formas 
de control y normas de acceso y uso de los recursos naturales basadas en su conocimiento ancestral, pero acordes a la legislación boliviana (Consejo Indígena del Pueblo Tacana y Wildlife Conservation Society 2005).

El territorio donde viven los tacanas forma parte del área de influencia del Parque Nacional (PN) Madidi - una de las mayores áreas protegidas del país (ca. 1.900 ha) con elevados registros de nuevas especies de plantas y animales. Esta ubicación propició que varios equipos de biólogos bolivianos interesados en realizar relevamientos de biodiversidad en el PN Madidi se acercaran a las comunidades tacanas para solicitar su colaboración en la realización de dichos inventarios. La relevancia del pueblo tacana en la realización de inventarios es especialmente importante, no solo por el conocimiento que pueden aportar, sino también por las potenciales implicaciones de estos inventarios en el uso de recursos (Bourdy et al. 2000, Moraes 2005, Paniagua-Zambrana et al. 2007, Campos Pastén \& Fernández Rodríguez 2019, Poma et al. 2019, Velasco Sarmiento 2019).

En este contexto, entre 2013 y 2015 se implementó el proyecto "Riqueza y etnobotánica de palmeras en Tumupasa." Las palmeras son uno de los grupos de plantas más importantes para las culturas de la región tropical debido a su gran diversidad de usos. Por ejemplo, en el oeste sudamericano el $63 \%$ de las 336 especies de palmeras conocidas es considerado útil, contando con un total de casi 2.400 usos (Moraes et al. 2015). Así mismo, casi un $60 \%$ de las cerca de 100 especies de palmeras nativas de Bolivia son utilizadas (Moraes 2014a). El proyecto de palmeras en Tumupasa, liderado por biólogos bolivianos, fue presentado a la directiva tacana, quien en 2012 lo respaldó y apoyó mediante la firma de un convenio suscrito entre el Consejo Indígena del Pueblo Tacana (CIPTA) y la UMSA. El objetivo del proyecto era documentar los usos y aplicaciones que los tacanas asignan a las especies nativas de palmeras. El proyecto se realizó mediante la aplicación de metodologías propias de la etnobotánica: entrevistas semiestructuradas, talleres participativos y acompañamientos con guías locales, entre los cuales fueron designados los conocedores representantes tacana (11 hombres y una mujer, Figura 1). 
Figura 1 : Sra. Casilda Cartagena con abanicos de Attalea princeps

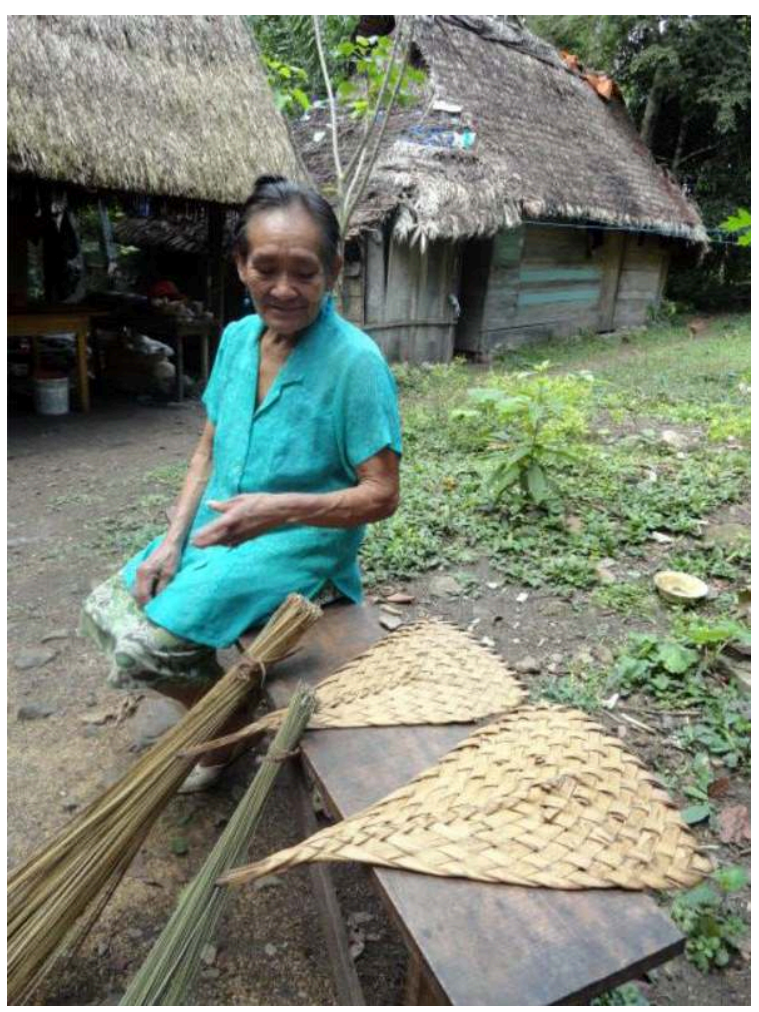

Foto: M. Moraes R.

20 Los resultados de este proyecto muestran que el 79\% de las 27 palmeras presentes en la zona son utilizadas útiles para los tacanas. Entre las 11 categorías de uso documentadas, las de mayor importancia local son la alimentación humana (29\%), los materiales para construcción (17\%), y la alimentación para la fauna (14\%). Las especies de palmeras más apreciadas por los tacanas son el motacú (Attalea princeps), el asaí (Euterpe precatoria), la jatata (Geonoma deversa), y el majillo (Oenocarpus mapora) (Cartagena et al. 2014). El asaí es la palmera con mayor número de registros en las entrevistas y representa en Tumupasa una especie multipropósito, de la cual aprovechan las hojas para techado, las raíces para medicinas, los frutos para bebidas y helados, y el cogollo para el palmito.

Más allá de los resultados científicos del proyecto, la dinámica generada en el trabajo de campo derivó en el intercambio de recomendaciones y la consideración de las prácticas de cosecha para una aproximación coherente al manejo sostenible de las palmeras. Estas consideraciones son especialmente importantes porque muchos de los pobladores que se asientan en el área de influencia de Tumupasa proceden de otras regiones del país y desconocen las características naturales del lugar, por lo que no aplican principios de una extracción responsable de recursos ni acorde con la planificación sostenible (Moraes 2014a, 2014b). Por tanto, un proyecto que se inició con el objetivo de conocer la diversidad biológica y sus usos locales, evolucionó hacia consideraciones de manejo con la población local, adoptando -por tanto- un enfoque etnobiológico. 


\section{De la antropología a la etnobiología: El conocimiento ecológico de los tsimane'}

Los tsimane' son un pueblo indígena que vive de la caza, la pesca, la recolección y la agricultura de subsistencia en un territorio que se extiende desde el piedemonte andino hasta las sabanas de Moxos, Departamento del Beni. Con una población de aproximadamente 14,000 personas distribuidas en unas 125 comunidades, este grupo étnico se ubica principalmente a lo largo de las riberas de los ríos Maniqui y Quiquibey, así como caminos de tala de madera (Reyes-García et al. 2014). Los relatos históricos los describen como una sociedad particularmente esquiva y muy móvil, que carece de un sistema jerárquico de autoridad (Huanca 2008). Hasta finales de la década de 1930, mantuvieron un patrón de asentamiento disperso y un estilo de vida autosuficiente, manteniendo solo contacto ocasional con otros grupos. A partir de la década de 1950, después del establecimiento de escuelas evangélicas en la zona, los tsimane' comenzaron a establecer asentamientos más permanentes. Las misiones tuvieron un impacto profundo en la cultura y el estilo de vida local a través de la introducción de prácticas cristianas (Huanca 2008). Después de la década de 1960, la construcción de nuevas carreteras, las diferentes oleadas de colonización planificada por el gobierno, el auge de la tala y las reformas de la tenencia de la tierra incrementaron el grado de exposición de los tsimane' con otros sectores de la sociedad boliviana, a menudo a través de la llegada de actores foráneos a su territorio (Reyes-García et al. 2014). Actualmente, mientras que algunos tsimane', particularmente aquellos que viven en áreas accesibles, incrementan sus interacciones con personas de otros grupos, otros se trasladaron a áreas más lejanas, como las aguas arriba de los ríos Maniqui y Sécure (Reyes-García et al. 2014).

Desde 1999, los cambios en la forma de vida de los tsimane' han sido profusamente documentados por un equipo antropológico interesado en el cambio cultural, en un proyecto conocido como "Tsimane' Amazonian Panel Study" (Godoy et al. 2009, Leonard et al. 2015, Reyes-García y Huanca 2015). En sus orígenes, esta investigación estaba orientada a estudiar cómo la modernización y la integración a la economía de mercado afectaban el conocimiento, la salud y la dieta tsimane' (p. ej., Reyes-García et al. 2019, Tanner et al. 2014), así como su uso de recursos naturales (Godoy et al. 2010) (Figura 2). Sin embargo, a lo largo de los años, la visión etnobiológica empezó a ganar peso en este proyecto con la realización de numerosos estudios sobre el conocimiento ecológico local y las relaciones de los tsimane' con su entorno. 
Figura 2. Ahumado de pescado en la cocina de un hogar tsimane'

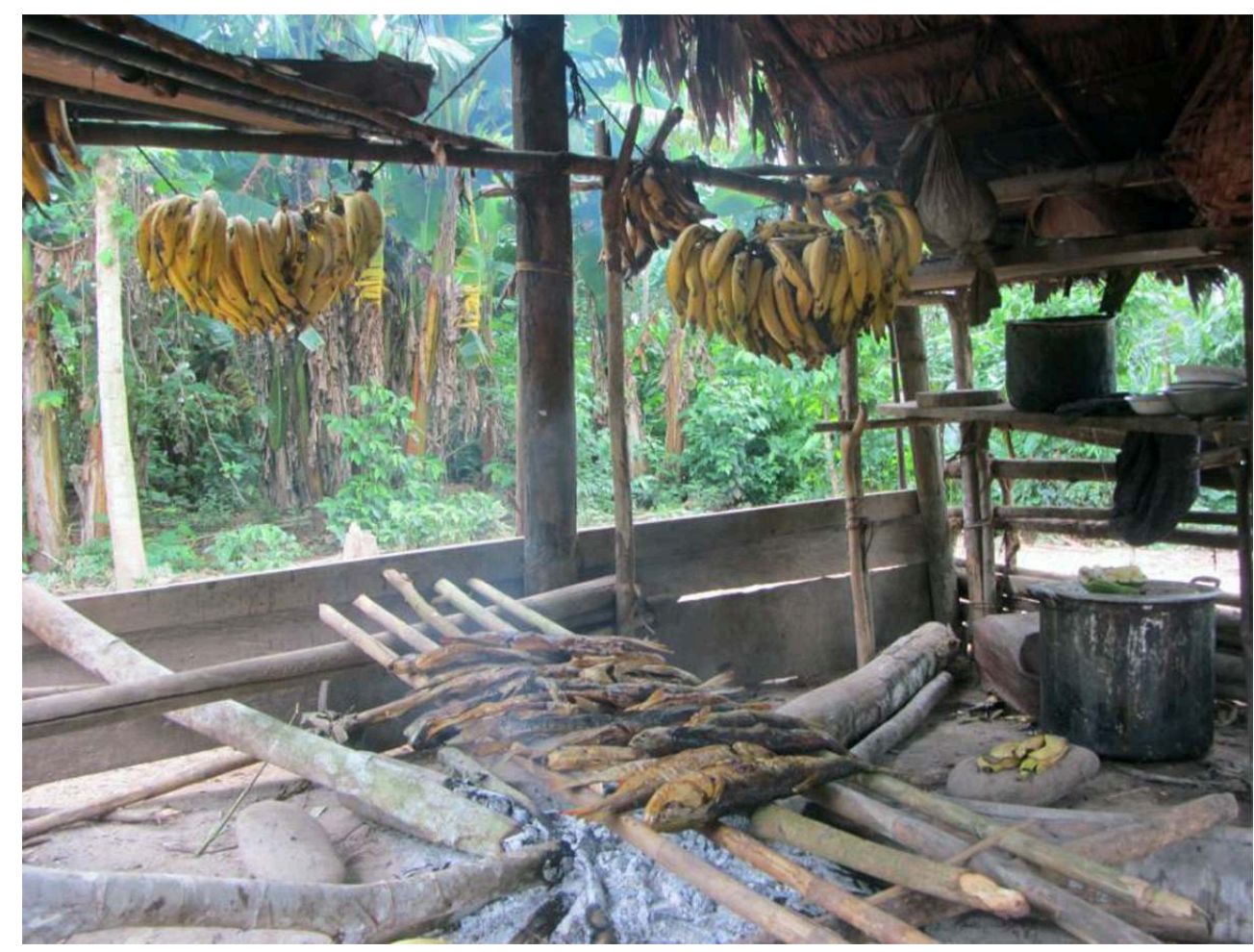

Foto: Á. Fernández- Llamazares

En su conjunto, este proyecto longitudinal ha puesto de relieve la importancia del conocimiento ecológico detentado por los tsimane' así como el proceso de cambio en este sistema de conocimientos. Por un lado, esta investigación muestra que la estrecha relación de los tsimane' con su entorno natural les ha permitido acumular una gran cantidad de conocimiento en diferentes dominios, incluyendo el conocimiento etnobotánico en general y de plantas medicinales en particular (Guèze et al. 2014, Reyes-García et al. 2006), pero también conocimiento de fauna, incluyendo el uso de diferentes técnicas y normas de caza (Apaza et al. 2002, 2003, Fernández-Llamazares et al. 2017, Luz et al. 2017), y conocimiento del clima (Fernández-Llamazares et al. 2015a) y del paisaje (Riu-Bosoms et al. 2015). Por otro lado, este proyecto también ha evidenciado el proceso de erosión del conocimiento ecológico local (Reyes-García et al. 2005, 2013), en gran medida causada por la falta de transmisión intergeneracional (FernándezLlamazares et al. 2015b). Finalmente, aunando la perspectiva biológica a la antropológica, este estudio también muestra que la pérdida de la cultura y forma de vida tradicional están estrechamente asociadas a la pérdida de diversidad biológica y la deforestación (Guèze et al. 2015, Paneque-Gálvez et al. 2018, Pérez-Llorente et al. 2013).

Es importante destacar que este proyecto, que se ha prolongado por dos décadas, ha contado con la colaboración con líderes comunitarios y se ha hecho en coordinación con el Gran Consejo Tsimane', la organización política de los tsimane' (FernándezLlamazares et al. 2020). Además, en el marco de esta investigación, se han llevado a cabo una serie de actividades educativas destinadas a mantener y revitalizar el conocimiento tsimane' y a divulgar los múltiples valores de este conocimiento. En concreto, a lo largo de los 20 años de investigación, se han utilizado una amplia gama de métodos y herramientas para valorar el conocimiento local incluyendo: 1) materiales educativos 
impresos, orales o visuales (p. ej., Nate et al. 2001, Reyes-García y Fernández-Llamazares 2019), 2) exposiciones para el gran público mostrando elementos de la cultura tsimane', y 3) talleres orientados a facilitar el empoderamiento de los tsimane' y valorizar su cultura (Fernández-Llamazares et al. 2020).

\section{Investigación participativa en etnobiología: La etnobotánica de los chácobos}

Los chácobos son un grupo de unas 1,000 personas pertenecientes al grupo lingüístico Pano y ubicados al norte de Bolivia. Los chácobos fueron visitados por investigadores por primera vez en 1911. Estos estudios documentan que, hasta finales de la década de 1890, los chácobos vivían como cazadores semi-nómadas y cultivaban yuca y maíz (Nordenskjöld 1922). Durante el auge del caucho a principios del siglo XX, los chácobos fueron forzados a dejar su territorio original. En 1953, misioneros del Instituto Lingüistico de Verano llegaron a las comunidades chácobo, donde permanecieron hasta 1980, generando profundos cambios en su cultura y tradiciones. Así, desde 1955, los chácobos fueron obligados a dejar su vida nómada y establecerse en comunidades permanentes para poder asistir a la escuela. Esto inició un proceso de cambio cultural que conllevó el abandono de su vestimenta y sus ceremonias tradicionales (Córdoba 2012).

Durante la segunda mitad del siglo XX, se produjo un renovado interés por la cultura chácobo (Bergeron 1998, Boom 1987, Haenke 1958, Prost 1960, Prost 1970). Aunque los estudios llevados a cabo tenían enfoques diferentes, todos registraron datos etnobotánicos y lingüísticos, señalando la pérdida del conocimiento tradicional chácobo. En base a esta información, el "Proyecto Etnobotánico Chácobo" fue concebido para indagar hasta qué punto el conocimiento etnobotánico documentado y la percepción de que este conocimiento se está perdiendo dependen de los entrevistadores y los métodos empleados (Figura 3). El proyecto fue desarrollado por 10 indígenas chácobos seleccionados por el pueblo, quienes fueron capacitados en el uso de técnicas de colecta de información etnobotánica incluyendo el uso de cuestionarios que fueron usados en las entrevistas y de técnicas de recolección y preservación de especímenes botánicos. También fueron capacitados para el registro fotográfico y en la elaboración y análisis de bases de datos. Se esperaba que el uso de una metodología $100 \%$ participativa ayudaría a documentar más exhaustivamente la pervivencia de usos mencionados en estudios previos. 
Figura 3 : Equipo del proyecto “Etnobotánica Chácobo"

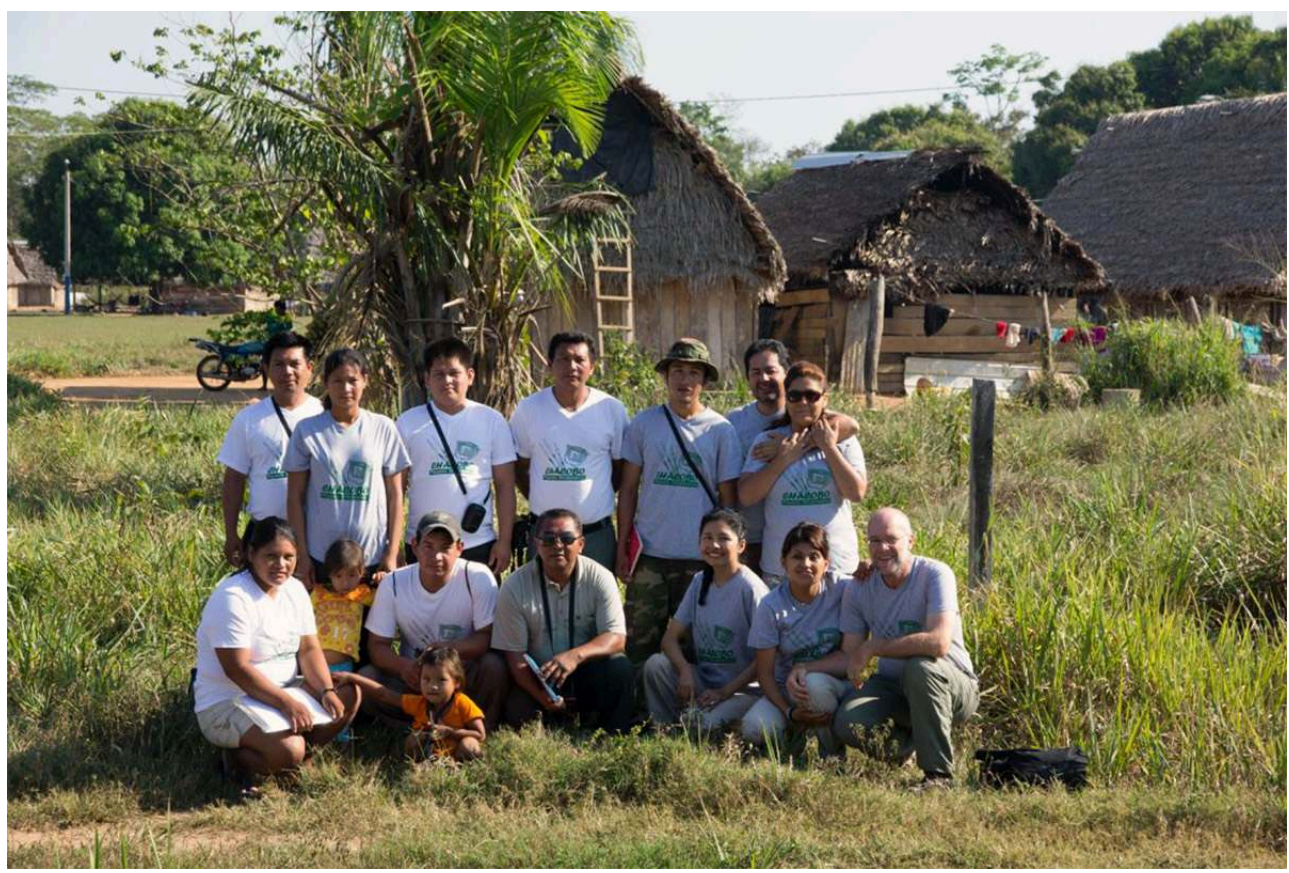

Foto: N. Paniagua-Zambrana

Entre noviembre de 2013 y mayo de 2015, los investigadores chácobos utilizaron listados libres para recoger información etnobotánica de 301 participantes, 150 mujeres y 151 hombres, casi la totalidad de la población chácobo adulta (>18 años). También colectaron más de 1,500 muestras de plantas útiles, que posteriormente fueron depositadas en el Herbario Nacional de Bolivia (LPB). El estudio documentó 331 especies de plantas útiles, muchas de las cuales se percibían en desuso (Paniagua-Zambrana et al. 2017).

29 En general, los resultados de este proyecto sugieren que el conocimiento chácobo solo se vio parcialmente afectado por los procesos de cambio y que, como resultado de estos procesos, diferentes dominios de conocimiento experimentaron diferentes tendencias (Paniagua-Zambrana et al. 2017). Es decir, los resultados llevan a pensar que la pérdida de conocimiento documentada en estudios previos puede ser parcialmente debida a los métodos de investigación empleados (Bussmann et al. 2018). En concreto, los resultados de este proyecto muestran que el número de entrevistas realizadas y el método de recogida de datos utilizado afectan la calidad de los datos (Paniagua-Zambrana et al. 2018a), un aporte metodológico importante en el campo de la etnobiología. Además, este proyecto también permitió demostrar la importancia de la identidad cultural y del conocimiento del entrevistador en la calidad de los datos. Aspectos como el idioma, la confianza, y la disponibilidad de tiempo, limitaciones comunes en estudios científicos, inciden en la calidad de la información etnobotánica recolectada (Paniagua-Zambrana et al. 2018b).

Un aspecto importante a destacar es que, en conformidad con el Protocolo de Nagoya, toda la información bibliográfica revisada en esta investigación fue traducida y devuelta a los chácobos bajo la forma de una compilación (Paniagua-Zambrana et al. 2014). La información etnobotánica recopilada durante el proyecto también fue devuelta en forma de bases de datos y en publicaciones que reconocen la autoría de 
todos los participantes y permiten proteger la propiedad intelectual de su conocimiento (Paniagua-Zambrana \& Bussmann 2017, Paniagua-Zambrana et al. 2017, Bussmann et al. 2018, Paniagua-Zambrana et al. 2018a,b). Este proyecto, por tanto, pone de relieve la importancia de la investigación participativa en etnobiología.

\section{Retos para el desarrollo de la etnobiología en Bolivia}

31 El desarrollo de la etnobiología en Bolivia se enfrenta a varios retos relacionados tanto con la evolución política del país como con la evolución de la disciplina en su contexto internacional. En esta sección, discutimos cuatro grandes retos a los que se enfrenta la etnobiología boliviana; i) la identificación de vacíos documentales y la creación de una línea de base, ii) el desarrollo metodológico con énfasis en la interdisciplinariedad, iii) la internacionalización; y iv) la generalización de la investigación participativa potenciando el diálogo de saberes.

\section{Identificación de vacíos documentales y creación de una línea de base}

Las últimas décadas han visto numerosos avances en el estudio de la diversidad biocultural de Bolivia. Pero, hasta ahora esta investigación ha tenido mayoritariamente un carácter disperso y unidisciplinario (Moraes et al. 2016), y no ha habido hasta ahora un esfuerzo de compilación documental. Por tanto, el primer reto para consolidar la etnobiología en Bolivia es la identificación de vacíos documentales y creación de una línea de base que permita orientar trabajos futuros.

La identificación del vacío documental debe iniciarse con la recopilación y catálogo de todo documento referente a investigaciones etnobiológicas realizadas en Bolivia de las últimas décadas y proveniente de cualquier disciplina. En el año 2016, investigadores de la UMSA y el ICTA-UAB crearon el primer respaldo bibliográfico etnobiológico de Bolivia, hospedado en la biblioteca del Herbario Nacional de Bolivia. Durante ese esfuerzo, se constató que muchos trabajos realizados en Bolivia no están disponibles físicamente en el país, por lo que el esfuerzo también debe enfocarse a conseguir la digitalización y/o repatriación de esos documentos a los que el público boliviano no tiene acceso.

La creación de un repositorio documental especializado a disposición de investigadores, estudiantes y comunidades locales de todo el país resulta imprescindible para la consolidación de la disciplina porque permitiría elaborar una línea de base en la que se puedan apoyar futuras priorizaciones de investigación. Por ejemplo, mientras que la etnobiología amazónica y andina siguen creciendo, son pocos los trabajos realizados en el Gran Chaco (ver Quiroga et al. 2012 o Scalise Sugiyama et al. 2020 para algunas excepciones). Así mismo, hay diferencias en el número de trabajos etnobiológicos realizados entre los diferentes pueblos indígenas. Por ejemplo, el conocimiento etnobotánico del pueblo quechua está muy bien documentado (Mathez-Stiefel et al. 2012a,b, Thomas et al. 2008, 2009b). En el contexto amazónico, mientras que pueblos como los tsimane' (Guèze et al. 2014, Reyes-García et al. 2006), los chácobos (PaniaguaZambrana et al. 2017), los lecos (Balslev et al. 2012), los trinitarios (Thomas et al. 2009c), los yuracarés (Hinojosa et al. 2001), o los tacanas (DeWalt et al. 1999, Hahn y Hissink 2000) cuentan con la documentación generada por estudios etnobiológicos, otros 
grupos, como los morés, los canichanas o los yaminahuas, no han recibido prácticamente ninguna atención desde la etnobiología. También conviene destacar que mientras que la etnobotánica y la etnofarmacología cuenta con varios estudios en Bolivia, subdisciplinas como la etnozoología, la etnomicología, o la etnoveterinaria todavía no se han iniciado en el país.

\section{Desarrollo metodológico con énfasis en la interdisciplinariedad}

La falta de consolidación de la disciplina en Bolivia se refleja también en la diversidad metodológica. En este sentido, los estudios existentes han utilizado metodologías dispares, por lo que es difícil establecer comparaciones entre ellos, o con los resultados de estudios globales. Mientras que este no es un problema único en Bolivia, la etnobiología boliviana -por su alto nivel de desarrollo y productividad- podría contribuir al desarrollo general de la disciplina proponiendo la sistematización de protocolos para la toma de datos. Este es un empeño ambicioso porque la etnobiología comparte intereses teóricos con varias disciplinas, incluyendo la antropología, la ecología, la geografía, la biología, la economía ecológica, la arqueología, o la agronomía, lo que ha llevado a la aplicación de un amplio abanico metodológico, incluyendo el uso de sistemas de información geográfica para entender la distribución espacial de fenómenos socio-ecológicos (p. ej., Paneque-Gálvez et al. 2018) o el uso de análisis de redes sociales para entender la transmisión de los conocimientos locales (p. ej., DíazReviriego et al. 2016). En este sentido, y dado que la etnobiología aspira a construir explicaciones holísticas sobre las inter-relaciones entre los seres humanos y la naturaleza, el desarrollo metodológico debe reconocer la importancia de la formación interdisciplinar.

\section{Internacionalización}

La consolidación de la etnobiología en Bolivia pasa también por promover su internacionalización. Científicos bolivianos han hecho importantes contribuciones a la etnobiología. Por ejemplo, equipos bolivianos han contribuido a entender mejor los patrones de uso de palmeras en los bosques tropicales lluviosos del noroeste de América del Sur (p. ej., Paniagua-Zambrana et al. 2007, Moraes et al. 2015). Pese a la importancia de la producción de los investigadores bolivianos, buena parte de la investigación etnobiológica en Bolivia que llega a ámbitos internacionales está en manos de equipos de cooperación científica o simplemente internacionales. Se estima que tan sólo un $4 \%$ de los estudios etnobiológicos publicados en revistas internacionales y realizados en Bolivia entre 1963 y 2012 fueron liderados por investigadores afiliados a instituciones bolivianas (Albuquerque et al. 2013). En este sentido, resulta crucial visibilizar la etnobiología boliviana a nivel internacional y promover el uso de nuevas herramientas metodológicas que permitan establecer redes con etnobiólogos trabajando con bases de datos a nivel transcultural (p. ej., Reyes-García et al. 2016), panamericano (p. ej. Cámara-Leret et al. 2014) o incluso global (p. ej., Koster et al. 2019). 


\section{Generalización de la investigación participativa potenciando el diálogo de saberes}

Uno de los grandes desafíos de la etnobiología es la adecuada inclusión de los pueblos indígenas y comunidades locales en su práctica. La aprobación del Protocolo de Nagoya (CBD 2011) regulando el acceso a los recursos genéticos y promoviendo la participación justa y equitativa en los beneficios que se deriven de su utilización provee las herramientas conceptuales para el diseño de una metodología participativa que incluya a los miembros de las comunidades locales en los estudios etnobotánicos. Dicho protocolo no solo considera el consentimiento previo e informado como una condición necesaria para el desarrollo de la investigación, sino que también propone la participación activa de los miembros de las comunidades indígenas y locales en el diseño y la realización de estos proyectos (Art. 12 y 23). Aunque muchos países, incluyendo Bolivia, aún no han reglamentado la implementación del Protocolo de Nagoya, los principios mencionados deberían ser considerados en todo el diseño y ejecución de las investigaciones etnobiológicas. Por otra parte, existen ya reglamentaciones (p. ej., Callaway 2017) y protocolos de ética (p.ej., el del ISE (https:// www.ethnobiology.net/wp-content/uploads/ISECodeofEthics_Spanish.pdf) o el de la SOLAE (http://asociacionetnobiologica.org.mx/aem/codigo-de-etica-de-solae) que rigen la realización de trabajos etnobiológicos regulando tanto el acercamiento a los pueblos indígenas y comunidades locales, como la protección de su patrimonio cultural e intelectual. En el contexto boliviano, un primer aporte importante para establecer lineamientos de trabajo colaborativo en las investigaciones etnobiológicas fue parte del manifiesto de las III Jornadas de Etnobiología realizadas en Santa Cruz de la Sierra (Moraes et al. 2019).

Es importante destacar que las consideraciones éticas plantean desafíos que van más allá de la aplicación de protocolos ya que afecta aspectos como la identidad y la representación de los pueblos indígenas. En ese sentido, más allá de cumplir con determinados códigos de ética en el proceso de investigación, la etnobiología se enfrenta al reto de establecer un posicionamiento ético adecuado a priori. Los pueblos indígenas y comunidades locales deben participar en la definición de los objetivos y las actividades de investigación tanto para asegurar el intercambio multidireccional de conocimientos entre investigadores, estudiantes, expertos locales, y otros grupos de interés, como para establecer acuerdos en la publicación de trabajos, consolidación del diálogo de saberes y resguardo de la propiedad intelectual e identidad cultural, que es reconocida como patrimonio intangible de la humanidad (Golan et al. 2019).

\section{Conclusión}

El creciente interés en la etnobiología refleja la actual preocupación social por el deterioro del medio ambiente y la pérdida de diversidad biocultural, así como por los impactos de procesos globales en los pueblos indígenas y sus territorios (p. ej., Fernández-Llamazares et al. 2018, Romero-Muñoz et al. 2019). La resolución de los acuciantes problemas socio-ecológicos requiere de una perspectiva interdisciplinar que logre integrar los intereses y conocimientos de las comunidades locales con el conocimiento científico. Por su carácter interdisciplinar y su perspectiva de análisis a varias escalas, la etnobiología puede contribuir a comprender e interpretar dichos 
problemas, ofreciendo numerosas oportunidades para su posterior solución. En Bolivia, la etnobiología tiene la posibilidad de proporcionar los elementos teóricos y metodológicos que permiten entender el papel de los pueblos indígenas y comunidades locales en la conservación del patrimonio biológico y cultural del país ante los grandes desafíos de nuestros tiempos. No obstante, y precisamente por su carácter interdisciplinar y su interés en abordar distintos saberes, la etnobiología se enfrenta a numerosos desafíos que solo pueden ser superados desde la perspectiva de la cooperación científica y el respeto a los derechos de los pueblos indígenas y comunidades locales.

Agradecemos a los miembros de los diferentes pueblos indígenas bolivianos, y en particular a los tacanas, los tsimane', y los chacobos, que a través de los años nos han abierto sus puertas y han compartido sus conocimientos e inquietudes con nosotros. También agradecemos los comentarios de I. Díaz-Reviriego y A.B. Junqueira a una versión previa de este artículo. Agradecemos a la Fundació Autònoma Solidaria (Universitat Autònoma de Barcelona) por el financiamiento del Fons de Solidaritat (convocatorias de 2015, 2017, y 2019) para la realización de las Jornadas de Etnobiología en Bolivia. También agradecemos al proyecto "Palmeras de Bolivia" del Herbario Nacional de Bolivia de la UMSA y al proyecto LICCI (FP7-771056-LICCI) del Consejo Europeo de Investigación por financiamiento parcial para la elaboración de este trabajo.

\section{BIBLIOGRAFÍA}

Alba J.J. 1993 - Tarifa \& Los Jampiris de Raqaypampa. Cochabamba: CENDA.

Albó X. 1972 - Dinámica en la estructura intercomunitaria de Jesús de Machaca. Mexico, Instituto Mexicano Indigenista.

Albó X. 1979 - ¿Khitipxtansa? ¿Quiénes somos? Identidad localista, étnica y clasista en los aymaras de hoy. América Indígena 39 (3) : 477-527.

Albuquerque U.P., Silva J.S., Campos J.L.A., Sousa R.S., Silva T.C. \& Alves R.R.N. 2013 - The current status of ethnobiological research in Latin America: Gaps and perspectives. Journal of Ethnobiology and Ethnomedicine 9 (1) : 72.

Apaza L., Wilkie D., Byron E., Huanca T., Leonard W., Pérez E., et al. 2002 - Meat prices influence the consumption of wildlife by the Tsimane' Amerindians of Bolivia. Oryx 36 (4) :382-388.

Apaza L., Godoy R., Wilkie D., Byron E., Huanca T., Leonard W.R., Pérez E., Reyes-García V. \& Vadez V. 2003 - Markets and the use of wild animals for traditional medicine: a case study among the Tsimane' Amerindians os the Bolivian rain forest. Journal of Ethnobiology 23 : 47-64.

Armstrong C.G. \& McAlvay A.C. 2019 - Introduction to Special Section on Action Ethnobiology. Journal of Ethnobiology 39 (1): 3 .

Atran S. 1985 - The Nature of Folk-Botanical Life Forms. American Anthropologist 87 (2) : 298-315. Balslev H., Paniagua-Zambrana N.Y., Blacutt Rivero E., Moraes R. M. \& Macía M.J. 2012 - Palmeras de los Lecos y sus usos. La Paz, Herbario Nacional de Bolivia. 
Barnadas J. 1976 - Los aymaras dentro de la sociedad boliviana. Cuadernos de Investigación CIPCA 12.

Barragán R. 2009 - Bolivia: Bridges and Chasms. In : A Companion to Latin American Anthropology : 32-55. Oxford, Blackwell Publishing Ltd.

Bergeron S. 1998 - El uso de las plantas por los chácobos (Alto Ivón, Beni, Bolivia). La Paz, Institut Françáis d'Etudes Andines.

Berkes F., Colding J. \& Folke C. 2000 - Rediscovery of Traditional Ecological Knowledge as Adaptive Management. Ecological Applications 10 (5) : 1251-1262.

Berlin B. 1976 - The concept of rank in ethnobiological classification: some evidence from Aguaruna folk botany. American Ethnologist 3 (3) : 381-399.

Berlin B., Breedlove D.E. \& Raven P.H. 1966 - Folk taxonomies and biological classification. Science 154 (3746) : 273-275.

Berlin B., Breedlove D. \& Raven P. 1973 - General Principles of Classification and Nomenclature in Folk Biology. American Anthropologist 75 (1) : 214-242.

Boillat S., Mathez-Stiefel S.L. \& Rist S. 2013 - Linking local knowledge, conservation practices and ecosystem diversity: Comparing two communities in the Tunari National Park (Bolivia).

Ethnobiology and Conservation 2 (2013). doi:10.15451/ec2013-8-2.8-1-28

Boom B.M. 1987 - Ethnobotany of the Chácobo Indians, Beni, Bolivia. Advances in economic botany. New York, New York Botanical Garden.

Bourdy, G. 2002 - Plantas del Chaco. Usos tradicionales Izoceño-Guaraní. La Paz, Fundación Kaa-Iya, IRD, CABI, WCS, Herbario Nacional de Bolivia.

Bourdy G., DeWalt S.J., Chávez de Michel L.R., Roca A., Deharo E., Muñoz V., et al. 2000 - Medicinal plants uses of the Tacana, an Amazonian Bolivian ethnic group. Journal of ethnopharmacology 70 (2) : 87-109.

Bouysse-Cassagne T. 1980 - La identidad aymara. Aproximación histórica. Siglo XV, siglo XVI. La Paz, HISBOL.

Brohan M. \& Herrera E. 2008 - Tabo Amapo, Alfredo. El eco de las voces olvidadas. Una autoetnografía y ethonistoria de los Cavineños de la Amazonia boliviana. Copenhaguen, IWGIA.

Bussmann R.W., Paniagua-Zambrana N.Y., Hart R.E., Moya Huanca A.L., Ortiz-Soria G., Ortiz-Vaca M., et al. 2018 - Research Methods Leading to a Perception of Knowledge Loss-One Century of Plant Use Documentation Among the chácobo in Bolivia. Economic Botany 72 (1) : 81-93.

Callaway E. 2017 - South Africa's San people issue ethics code to scientists. Nature 543 : 475-476.

Cámara-Leret R., Paniagua-Zambrana N., Balslev H. \& Macía M.J. 2014 - Ethnobotanical Knowledge Is Vastly Under-Documented in Northwestern South America. PLoS ONE 9 (1) : e85794.

Campos Pasten P.J. \& Fernández Rodríguez V.M. 2019 - Uso cinegético y aprovechamiento en el Jardín Botánico y área de influencia en Tumupasa. Programa Integral Biológico-Turístico Jardín Botánico de Tumupasa, Universidad Mayor de San Andrés, La Paz. 117 p.

Cárdenas M. 1969 - Manual de plantas económicas de Bolivia. Cochabamba, Los Amigos del Libro.

Cartagena T., Pardo Apana A., Terrazas Achimo J., Medina Gonzales N., Cartagena Cuajera C., Marupa Amutari L., et al. 2014 - Palmeras útiles de Tumupasa. In : Moraes M. R. (Ed.), Palmeras de Tumupasa en La Paz, Bolivia : 19-28. La Paz, Universidad Mayor de San Andres. 
Concardo Morales R. 1978 - Reflexiones acerca del eco-sistema vertical andino. Avances. Revista de Ciencias Socialdes $1: 65-74$.

Conklin H.C. 1954 - An ethnoecological approach to shifing agriculture. Transactions of the New York Academy of Sciences 17 (2 Series II) : 133-142.

Convenio sobre la Diversidad Biológica-CBD 2011 - Protocolo de Nagoya sobre Acceso a los recursos genéticos y participación justa y equitativa en los beneficios que se deriven de su utilización al Convenio sobre la Diversidad Biológica: Texto y anexo. Secretaría del Convenio sobre la Diversidad Biológica. Programa de las Naciones Unidas para el Medio Ambiente. Montreal, Canadá, $26 \mathrm{p}$

Consejo Indígena del Pueblo Tacana, Wildlife Conservation Society 2005 - Estrategia de desarrollo sostenible de la TCO-Tacana con base en el manejo de los recursos naturales. La Paz.

Córdoba L.I. 2012 - Misiones-patrones e indígenas-siringueros: el caucho entre los chacobos del Beni (siglo xx). Boletína Americanista LXII-2 (65) : 85-106.

De Lucca M. \& Zalles J. 1992 - Flora medicinal boliviana, diccionario enciclopédico. La Paz, SEAPAS, Los Amigos del Libro.

Denevan W.M. 1966 - The Aboriginal Cultural Geography of the Llanos de Mojos of Bolivia. Berkeley, University of California Press.

DeWalt S.J., Bourdy G., Chávez De Michel L.R. \& Quenevo C. 1999 - Ethnobotany of the Tacana: Quantitative inventories of two permanent plots of northwestern Bolivia. Economic Botany 53 (3) : 237-260.

Díaz-Reviriego I., González-Segura L., Fernández-Llamazares Á., Howard P.L., Molina J.L. \& ReyesGarcía V. 2016 - Social organization influences the exchange and species richness of medicinal plants in amazonian homegardens. Ecology and Society 21 (1).

Díez Astete A. 2011 - Compendio de etnias indígenas y ecorregiones: Amazónica, Oriente y Chaco. La Paz, Centro de Servicios Agropecuarios y Socio-Comunitarios. Plural editores.

Ellen R. 2004 - From Ethno-Science to Science, or "What the Indigenous Knowledge Debate Tells Us about How Scientists Define Their Project." Journal of Cognition and Culture 4 (3-4) : 409-450.

Erickson C. 2011 - Agency, Causeways, Canals, and the Landscapes of Everyday Life in the Bolivian Amazon. In : Snead J., Erickson C. \& Darling A. (Ed.) Landscapes of movement: Trails, paths, and roads in anthropological perspective : 204-231. Philadelphia, PennMuseum Press and the University of Pennsylvania Press.

Fernández-Llamazares Á., Méndez-López M.E., Díaz-Reviriego I., McBride M., Pyhälä A., RosellMelé A. \& Reyes-García V. 2015a - Links between scientific framings and local perceptions of climate change in an indigenous society. Climatic Change $131: 307-320$.

Fernández-Llamazares Á., Díaz-Reviriego I., Luz A.C., Cabeza M., Pyhälä A. \& Reyes-García V. 2015 b - Rapid ecosystem change challenges the adaptive capacity of local environmental knowledge. Global Environmental Change 31 : 272-284.

Fernández-Llamazares Á., Díaz-Reviriego I. \& Reyes-García V. 2017 - Defaunation Through the Eyes of the Tsimane'. In : Hunter-gatherers in a Changing World : 77-90. Springer International Publishing.

Fernández-Llamazares Á., Helle J., Eklund J., Balmford A., Moraes R. M., Reyes-García V. \& Cabeza M. 2018 - New law puts Bolivian biodiversity hotspot on road to deforestation. Current Biology 28 (1) : R15-R16. doi: 10.1016/j.cub.2017.11.013 
Fernández-Llamazares Á., Benyei P., Junqueira A.B., Reyes-García V. 2020 - Participation in Biocultural Diversity Conservation: Insights from five Amazonian examples. In : Baldauf C.P. (Ed.), Participatory Biodiversity Conservation - Concepts, experiences, and perspectives. Switzerland, Springer Nature.

Gandarillas H. 1979 - Genética y origen de la quinua. In : Tapia M.E. (Ed.), Quinua y Kanihua, Cultivos Andinos : 45-64). Bogotá, IICA.

Girault L. 1987 - Kallawaya: curanderos itinerantes de los Andes, investigacion sobre practicas medicinales y magicas. Bolivia, Unicef.

Godoy R., Reyes-García V., Byron E., Leonard W.R. \& Vadez V. 2005 - The effect of market economies on the well-being of indigenous peoples and on their use of natural resources. Annual Review of Anthropology $34: 121-138$.

Godoy R., Reyes-García V., Gravlee C.C., Huanca T., Leonard W.R., McDade T.W. \& Tanner S. 2009 Moving beyond a snapshot to understand changes in the well-being of native amazonians: Panel evidence (2002-2006) from Bolivia. Current Anthropology 50 (4) : 563-573.

Godoy R., Undurraga E.A., Wilkie D., Reyes-García V., Huanca T., Leonard W.R., et al. 2010 - The effect of wealth and real income on wildlife consumption among native Amazonians in Bolivia: Estimates of annual trends with longitudinal household data (2002-2006). Animal Conservation 13 (3) : 265-274.

Golan J., Athayde S., Olson E.A. \& McAlvay A. 2019 - Intellectual Property Rights and Ethnobiology: An Update on Posey's Call to Action. Journal of Ethnobiology 39 : 90-109.

Guèze M., Luz A.C., Paneque-Gálvez J., Macía M.J., Orta-Martínez M., Pino J. \& Reyes-García V. 2014 - Are Ecologically Important Tree Species the Most Useful? A Case Study from Indigenous People in the Bolivian Amazon. Economic Botany 68 (1) : 1-15.

Guèze M., Luz A.C., Paneque-Gálvez J., Macía M.J., Orta-Martínez M., Pino J. \& Reyes-García V. 2015 - Shifts in indigenous culture relate to forest tree diversity: A case study from the Tsimane', Bolivian Amazon. Biological Conservation 186 : 251-259.

Haenke W. 1958 - The Chácobo in Bolivia. Ethnos 23 : 100-125.

Hahn A. \& Hissink K. 2000 - Los tacana. Datos sobre la historia de su civilización. La Paz, APCOB, Plural editores.

Harris O. 1978 - From Asymmetry to Triangle: Symbolic Transformations in Northern Potosi. In : Murra J., Revel J. \& Watchtel N. (Ed.), Anthropological History of Andean Polities : 260-280. Cambridge, Cambridge University Press.

Hensen I. 1992 - La Flora de la Comunidad de Chorojo. Su uso, taxonomía cientifica y vernacular. Cochabamba, AGRUCO.

Hinojosa, I. 1991 - Plantas utilizadas por los Mosetenes de Santa Ana (Alto Beni-Depto. La Paz). Universidad Mayor de San Andrés, La Paz.

Hinojosa I., Uzquiano E., Flores J. \& Chávez N. 2001 - Los Yuracaré: su conocimiento, experiencia y la utilización de recursos vegetales en el Rio Chapare. La Paz, Instituto de Ecología - Fondo Nacional de Medio Ambiente.

Huanca T. 2008. Tsimane' Oral Tradition, Landscape, and Identity in Tropical Forest. La Paz, Imprenta Wagui.

Hunn E. 1982 - The Utilitarian Factor in Folk Biological Classification. American Anthropologist 84 (4) : 830-847. 
Hunn E. 2007 - Ethnobiology in four phases. Journal of Ethnobiology 27 : 1-10.

Huntington H.P. 2000 - Using traditional ecological knowledge in science: Methods and applications. Ecological Applications 10 (5) : 127-1274.

Ibisch P.L. \& Mérida G. (Ed.) 2003 - Biodiversidad: La riqueza de Bolivia. Editorial FAN.

IPBES 2019 - Summary for policymakers of the global assessment report on biodiversity and ecosystem services of the Intergovernmental Science-Policy Platform on Biodiversity and Ecosystem Services. Díaz S., Settele J., Brondízio E.S., Ngo H.T., Guèze M., Agard J., et al., (Ed.). Bonn, IPBES secretariat.

Koster J., McElreath R., Hill K., Yu D., Shepard G., van Vliet N., et al. 2019 - The Life History of Human Foraging: Cross-Cultural and Individual Variation. bioRxiv 574483. doi:10.1101/574483

Laing A.F. 2015 - Resource Sovereignties in Bolivia: Re-Conceptualising the Relationship between Indigenous Identities and the Environment during the TIPNIS Conflict. Bulletin of Latin American Research 34 (2) : 149-166.

Langlie B.S., Harstorf C.A., Bruno M.C., Bermann M., Bonzani R.M. \& Castellón Condarco W. 2011 Diversity in Andean Chenopodium Domestication: Describing a new morphological type from La Barca, Bolivia 1300-1250 B.C. Journal of Ethnobiology 31 : 72-88.

Larson B. 2019 - Revisiting bolivian studies: Reflections on theoryscholarship, and activism since 1980. Latin American Research Review 54 (2) : 294-309.

Lehm Z. 2016 - Sistematización de la historia del gobierno en la región tacana. La Paz, Wildlife Conservation Society Bolivia - Consejo Indígena del Pueblo Tacana, MacArthur \& Moore Foundation.

Leonard W.R., Reyes-García V., Tanner S., Rosinger A., Schultz A., Vadez V., et al. 2015 - The Tsimane' Amazonian Panel Study (TAPS): Nine years (2002-2010) of annual data available to the public. Economics and Human Biology 19 : 51-61.

Lombardo U., Iriarte J., Hilbert L., Ruiz-Pérez J., Capriles J.M. \& Veit H. 2020 - Early Holocene crop cultivation and landscape modification in Amazonia. Nature 1-4. doi:10.1038/s41586-020-2162-7

Loza-Balsa G. 1995 - Enciclopedia de la medicina Aymara. La Paz, OPS, OMS.

Luz A.C., Paneque-Gálvez J., Guèze M., Pino J., Macía M.J., Orta-Martínez M., Reyes-García V. 2017 - Continuity and change in hunting behaviour among contemporary indigenous peoples. Biological Conservation 209 : 17-26.

Macía M.J., García E. \& Vidaurre P.J. 2005 - An ethnobotanical survey of medicinal plants commercialized in the markets of la Paz and El Alto, Bolivia. Journal of Ethnopharmacology 97 (2) : 337-350.

Mann C.C. 2008 - Ancient earthmovers of the Amazon. Science 321 (5893) : 1148-1152.

Martius C. 1843 - Palmetum orbignyanum. In : Orbigny A. d' (Ed.), Voyage dans l'Amérique méridionale : v. $7: 1-140)$. Paris.

Mathez-Stiefel S.L., Vandebroek I. \& Rist S. 2012a - Can Andean medicine coexist with biomedical healthcare? A comparison of two rural communities in Peru and Bolivia. J Ethnobiology Ethnomedicine $8: 26$.

Mathez-Stiefel S.L., Brandt R., Lachmuth S. \& Rist S. 2012b - Are the young less knowledgeable? Local Knowledge of Natural Remedies in the Andean Highlands. Human Ecology 40 : 909-930.

Milton K. 2010 - Ecologies: anthropology, culture and the environment. International Social Science Journal 49 (154) : 477-495. 
Moraes R. M. 2005 - Prefacio. Número especial "Inventario florístico de la Región Madidi." Ecología en Bolivia 40 (3) : iii-iv.

Moraes R. M. 2014a - Palmeras útiles de Bolivia - Las especies mayormente aprovechadas para diferentes fines y aplicaciones. La Paz, Herbario Nacional de Bolivia, Universidad Mayor de San Andrés, Plural editores.

Moraes R. M. 2014b - Palmeras de Tumupasa en La Paz, Bolivia. La Paz, Herbario Nacional de Bolivia, Instituto de Ecología, Carrera de Biología, Universidad Mayor de San Andrés.

Moraes R. M., Arce, J., \& Mariaca, J. 1996 - Informe nacional de recursos fitogenéticos. In : IV Conferencia Internacional y Programa de Recursos Fitogenéticos. La Paz, Ministerio de Desarrollo Sostenible y Medio Ambiente.

Moraes R. M., Paniagua-Zambrana N.Y., Camara-Leret R., Balsev H., Macía M.J. 2015 - Palmas útiles de Bolivia, Colombia, Ecuador y Perú. In : Balslev H., Macía M.J. \& Navarrete H. (Ed.), Cosecha de Palmas en el Noroeste de Suramérica: Bases Científicas para su Manejo y Conservación : 87-102. Quito, Pontificia Universidad Católica del Ecuador.

Moraes R. M., Reyes-García V., Gueze M., Díaz-Reviriego I., Fernández-Llamazares Á. 2016 - Un impulso a la investigación etnoecológica en Bolivia. Ecología en Bolivia 51 (1) : 1-3.

Moraes R. M., Vargas V., Serrudo V., Blanco L. \& Palma V. 2019 - Lineamientos de colaboración de investigaciones etnobiológicas y etnoecológicas en Bolivia. Santa Cruz de la Sierra, Bolivia.

Nate A., Ista D. \& Reyes-García V. 2001 - Plantas Útiles y su Aprovechamiento en la Comunidad tsimane' de Yaranda. Santa Cruz de la Sierra, CIDOB-DFID.

Nordenskjöld E. 1922 - Indianer und Weisse im Nordosten Boliviens. Stuttgart, Strecker und Schröder. Nordenskjöld E. 1924 - The Ethnography of South America as Seen from Mojos in Bolivia (Number 3). Goteborg, Comparative Ethnographic Studies.

Orlove B.S. \& Godoy R. 1986 - Sectoral fallowing systems in the Central Andes. Journal of Ethnobiology 6 :169-204.

Paneque-Gálvez J., Pérez-Llorente I., Luz A.C., Guèze M., Mas J.F., Macía M.J., et al. 2018 - High overlap between traditional ecological knowledge and forest conservation found in the Bolivian Amazon. Ambio 47 : 908-923.

Paniagua-Zambrana N.Y., Byg A., Svenning J.C., Moraes R. M., Grandez C. \& Balslev H. 2007 Diversity of palm uses in the western Amazon. Biodiversity and Conservation 16 (10) : 2771-2787.

Paniagua-Zambrana N., Bussmann R.W., Vega C. \& Tellez C. (Ed.) 2014 - Los Chacobo y su historia en el siglo XX. Herbario Nacional de Bolivia, William L. Brown Center, Missouri Botanical Garden. Graficart, Trujillo, Perú, 390 p.

Paniagua-Zambrana N., Bussmann R.W. (Ed.) 2017 - La etnobotánica de los chácobo en el siglo XXI. Herbario Nacional de Bolivia, Instituto de Ecología, Universidad Mayor de San Andrés, William L. Brown Center. Missouri Botanical Garden. Graficart, Trujillo, Perú, 145 p.

Paniagua-Zambrana N.Y., Bussmann R.W., Hart R.E., Moya Huanca A.L., Ortiz Soria G., Ortiz Vaca M., et al. 2017 - Traditional knowledge hiding in plain sight - twenty-first century ethnobotany of the Chácobo in Beni, Bolivia. Journal of Ethnobiology and Ethnomedicine 13 (1) : 57.

Paniagua-Zambrana N.Y., Bussmann R.W., Hart R.E., Huanca A.L.M., Soria G.O., Vaca,M.O., et al. 2018 a - To list or not to list? the value and detriment of freelisting in ethnobotanical studies. Nature Plants 4 (4) : 201-204. 
Paniagua-Zambrana N.Y., Bussmann R.W., Hart R.E., Moya-Huanca A.L., Ortiz-Soria G., Ortiz-Vaca M., et al. 2018b - Who should conduct ethnobotanical studies? Effects of different interviewers in the case of the Chácobo Ethnobotany project, Beni, Bolivia. Journal of Ethnobiology and Ethnomedicine 14 (1) : 9.

Pérez-Llorente I., Paneque-Gálvez J., Luz A.C., Macía M.J., Guèze M., Domínguez-Gómez J.A., \& Reyes-García V. 2013 - Changing indigenous cultures, economies and landscapes: The case of the Tsimane', Bolivian Amazon. Landscape and Urban Planning 120 : 147-157.

Pestalozzi H. \& Torrez M. 1998 - Flora ilustrada altoandina. La relación entre hombre, planta y medio ambiente en el ayllu Majasaya Mujlli (Prov. Tapacarí, Depto. Cochabamba, Bolivia). Cochabamba, Herbario Forestal Nacional Martín Cárdenas.

Pitcher T.J. 2001 - Fisheries Managed to Rebuild Ecosystems? Reconstructing the Past to Salvage the Future. Ecological Applications 11 (2) : 601-611.

Poma A., Palabral O. \& Barboza-Marquez K. 2019 - Especies maderables y comercio del Jardín Botánico y área de influencia en Tumupasa. Programa Integral Biológico-Turístico Jardín Botánico de Tumupasa, Universidad Mayor de San Andrés, La Paz, 130 p.

Posey D.A. 1985 - Indigenous management of tropical forest ecosystems: the case of the Kayapó indians of the Brazilian Amazon. Agroforestry Systems 3 (2) : 139-158.

Prestes-Carneiro G. \& Béarez P. 2017 - Swamp-eel (Synbranchus spp.) fishing in Amazonia from pre-Columbian to present times. Journal of Ethnobiology 37 : 380-397.

Prost G. 1960 - Notas linguísticas de Bolivia. No. II: Phonemas de la lengua chácobo. La Paz, Instituto Linguístico de Verano.

Prost M. 1970 - Costumbres, habilidades y cuadro de vida entre los chácobos. La Paz, Instituto Linguístico de Verano.

Quiroga R., Meneses L. \& Bussmann R.W. 2012 - Medicinal ethnobotany in Huacareta (Chuquisaca, Bolivia). Journal of Ethnobiology and Ethnomedicine 8 (1) : 29.

Rea J. 1992 - Raíces andinas. In : J. Hernández Bermejo J.E. \& León J. (Ed.), Cultivos Marginados, otra Perspectiva de 1492 : 163-177. Roma, FAO.

Reyes-García V., Godoy R., Vadez V., Apaza L., Byron E., Huanca T., et al. 2003 - Ethnobotanical knowledge shared widely among Tsimane' Amerindians, Bolivia. Science 299 (5613) : 1707.

Reyes-García V., Vadez V., Byron E., Apaza L., Leonard W.R., Perez E. \& Wilkie,D. 2005 - Market economy and the loss of folk knowledge of plant uses: Estimates from the Tsimane' of the Bolivian Amazon. Current Anthropology 46 (4) : 651-656

Reyes-García V., Huanca T., Vadez V., Leonard W. \& Wilkie D. 2006 - Cultural, practical, and economic value of wild plants: A quantitative study in the Bolivian Amazon. Economic Botany 60 (1) : 62-74.

Reyes-García V. \& Martí Sanz N. 2007 - Etnoecología: punto de encuentro entre naturaleza y cultura. Ecosistemas: Revista científica y técnica de ecología y medio ambiente 16 (3) : 5.

Reyes-García V., Luz A.C., Gueze M., Paneque-Gálvez J., Macía M.J., Orta-Martínez M. \& Pino J. 2013 - Secular trends on traditional ecological knowledge: An analysis of changes in different domains of knowledge among Tsimane' men. Learning and Individual Differences 27 : 206-212.

Reyes-García V., Paneque-Gálvez J., Bottazzi P., Luz A.C., Gueze M., Macía M.J., et al. 2014 Indigenous land reconfiguration and fragmented institutions: A historical political ecology of Tsimane' lands (Bolivian Amazon). Journal of Rural Studies 34 : 282-291. 
Reyes-García V. \& Huanca T. 2015 - Cambio global, cambio local. La sociedad tsimane' ante la globalización. Barcelona, Icaria and Institut Català d'Antropologia.

Reyes-García V., Guèze M., Díaz-Reviriego I., Duda R., Fernández-Llamazares Á., Gallois S., et al. 2016 - The adaptive nature of culture. A cross-cultural analysis of the returns of local environmental knowledge in three indigenous societies. Current anthropology 57 (6) : 761-784.

Reyes-García V. \& Fernández-Llamazares Á. 2019 - Sing to Learn: The Role of Songs in the Transmission of Indigenous Knowledge among the Tsimane' of Bolivian Amazonia. Journal of Ethnobiology 39 (3) : 460-471.

Reyes-García V., Powell B., Díaz-Reviriego I., Fernández-Llamazares Á., Gallois S. \& Gueze M. 2019 - Dietary transitions among three contemporary hunter-gatherers across the tropics. Food Security 11 (1) : 109-122.

Reyes-García V., Fernández-Llamazares Á., Aumeeruddy-Thomas Y., Benyei P., Bussmann R., Diamond S., García-del-Amo D., Guadilla-Sáez S., Hanazaki N., Kosoy N., Lavides M., Luz A.C., McElwee P., Meresky V.J., Newberry T., Molnár Z., Ruiz-Mallén I., Salpeteur M., Wymdhan F., Zorondo-Rodriguez F. \& Brondizio E.S. 2022 - Recognizing Indigenous peoples' and local communities' rights and agency in the post-2020 Biodiversity Agenda. Ambio 51 : 84-92 (2022) https://doi.org/10.1007/s13280-021-01561-7

Riu-Bosoms C., Vidal T., Duane A., Fernández-Llamazares Á., Gueze M., Luz A.C., et al. 2015 Exploring Indigenous Landscape Classification across Different Dimensions: A Case Study from the Bolivian Amazon. Landscape Research 40 (3) : 318-337.

Rivera Cusicanqui S. 1999 - Sendas y senderos de la ciencia social Andina. Dispositio 24 (5) : 149-169.

Romero-Muñoz A., Fernández-Llamazares Á., Moraes R. M., Larrea-Alcázar D.M. \& Wordley C.F.R. 2019 - A pivotal year for Bolivian conservation policy. Nature Ecology and Evolution 3 : 866-869.

Saignes T. 1986 - En Busa del doblamiento étnico de los Andes bolivianos (Siglos XV y XVI). La Paz, Museo Nacional de Etnografía y Folklore.

Salomon F. 1985 - The historical development of Andean ethnology. Mountain Research \& Development 5 (1) : 79-98.

Sarmiento F.O. 2010 - The lapwing in andean ethnoecology: Proxy for landscape transformation. Geographical Review 100 (2) : 229-245.

Scalise Sugiyama M., Mendoza M. \& Quiroz I. 2020 - Ethnobotanical Knowledge Encoded in Weenhayek Oral Tradition. Journal of Ethnobiology 40 : 39-55.

Sillitoe P. 2006 - Ethnobiology and applied anthropology: Rapprochement of the academic with the practical. Journal of the Royal Anthropological Institute 12(s1) : S119-S142.

Tanner S., Leonard W.R. \& Reyes-García V. 2014 - The consequences of linear growth stunting: Influence on body composition among youth in the Bolivian Amazon. American Journal of Physical Anthropology 153 (1) : 92-102.

Tejeiro J. 2010 - Regionalización y diversidad étnica cultural en las tierras bajas y sectores del subandino amazónico y platense de Bolivia (Plural Edi.). La Paz.

Thomas E. 2009 - Quantitative Ethnobotany in Bolivia Knowledge, Use and Diversity of Plants in Quechua, Yuracaré and Trinitario Communities from the Andes and Amazon. VDM Verlag Dr. Müller. 
Thomas E., Vandebroek I., Goetghebeur P., Sanca S., Arrázola S. \& Van Damme P. 2008 - The Relationship Between Plant Use and Plant Diversity in the Bolivian Andes, with Special Reference to Medicinal Plant Use. Human Ecology $36: 861-879$.

Thomas E., Vandebroek I. \& Van Damme P. 2009a - Valuation of forests and plant species in indigenous territory and National Park Isiboro-Sécure, Bolivia. Economic Botany 63 (3) : 229-241.

Thomas E., Vandebroek I., Sanca S. and Van Damme P. 2009b - Cultural significance of medicinal plant families and species among Quechua farmers in Apillapampa, Bolivia. Journal of Ethnopharmacology $122: 60-67$.

Thomas E., Vandebroek I., Van Damme P., Semo L., Noza Z. 2009c - Susto Etiology and Treatment According to Bolivian Trinitario People: A "Masters of the Animal Species" Phenomenon. Medical Anthropology Quarterly 23 : 298-319.

Toledo M. 1996 - Etnobotánica de los chiquitanos de la región de Lomerío, en Santa Cruz, Bolivia. Ecología en Bolivia 27 : 31-42.

Toledo V.M., Stepp J.R., Wyndham F.S. \& Zarger R.K. 2002 - Ethnoecology: a conceptual framework for the study of indigenous knowledge of nature. In : International congress of ethnobiology; Ethnobiology and biocultural diversity : 511-522.

Townsend W. 2000 - The sustainability of subsistence hunting by the Sirionó Indians of Bolivia. In : Robinson J. \& Bennett E. (Ed.), Hunting for Sustainability in Tropical Forests : 267-281. New York, Columbia University Press.

Townsend W.R. \& Rumiz D.I. 2004 - Reflexiones sobre la posibilidad de manejo de fauna silvestre en la tierras bajas de Bolivia: experiencias comunitarias. Revista boliviana de ecología y conservación ambiental $16: 61-72$.

Turner K. L. 2019 - Biocultural Diversity, Campesino Kitchens, and Globalization: Ethnobiological Perspectives on Dietary Change in Southern Bolivia. Journal of Ethnobiology $39: 110-130$.

Vandebroek I., Calewaert J.B., De Jonckheere S., Sanca S., Semo L., Van Damme P., et al. 2004 - Use of medicinal plants and pharmaceuticals by indigenous communities in the Bolivian Andes and Amazon. Bulletin of the World Health Organization 82 (4) : 243-250.

Vargas L. 1996 - Etnobotánica de las plantas medicinales de los Mosetenes que viven en la Comunidad de Muchanes. Universidad Mayor de San Andrés, La Paz.

Velasco Sarmiento J.R. 2019 - Potencial piscícola: Implementación de centro productivo tacana en Ixiamas y capacitación local en San Buenaventura. Programa Integral Biológico-Turístico Jardín Botánico de Tumupasa, Universidad Mayor de San Andrés, La Paz, 50 p.

Vidaurre P., Paniagua-Zambrana N.Y., Moraes R. M. 2006 - Plantas Medicinales de los Andes de Bolivia. In : Moraes M. R, øllgaard B., Kvist L.P., Borchsenius F. \& Balslev H. (Ed.), Botánica Económica de los andes Centrales : 268-284. La Paz, Universidad Mayor de San Andres.

Wachtel N. 1990 - Le Retour des ancêtres. Les Indiens Urus de Bolivie XXe-XVIe siècle. Essai d'histoire régressive. Paris, Gallimard.

Zimmerer K. S. 1994 - Local soil knowledge: Answering basic questions in highland Bolivia. Journal of Soil and Water Conservation 49 (1) : 29-34. 


\section{NOTAS}

1. En este contexto, el conocimiento ecológico local se define como el conjunto de relaciones entre el kosmos (las creencias y representaciones simbólicas), el corpus (el conocimiento ambiental), y la praxis (los comportamientos que llevan a la apropiación de la naturaleza) de un grupo cultural en relación a su ambiente local (Toledo et al. 2002).

\section{RESÚMENES}

La etnobiología estudia las relaciones que diferentes sociedades entablan con la naturaleza analizando conocimientos, usos y percepciones. Bolivia es un país con una gran diversidad biológica y cultural. En las últimas dos décadas, Bolivia ha iniciado un proceso político de defensa de la identidad y la gobernanza indígena que incluye la revalorización del conocimiento ecológico local. Esta coyuntura proporciona una oportunidad excelente para el desarrollo y la consolidación de la investigación etnobiológica en el país. En este artículo, documentamos los precursores biológicos y antropológicos de la etnobiología boliviana y analizamos la evolución de la disciplina usando tres estudios de caso emblemáticos: los dos primeros muestran la confluencia de un proyecto de carácter biológico y uno de carácter antropológico hacia la etnobiología, y el tercero ejemplifica los beneficios del enfoque participativo. La última sección aborda algunos de los grandes retos de la etnobiología en Bolivia, centrándose en tres aspectos que permitirían asentar unas bases fuertes para su desarrollo: i) la identificación de vacíos documentales y la creación de una línea de base; ii) el desarrollo metodológico con énfasis en la interdisciplinariedad, iii) la internacionalización; y iv) la generalización de la investigación participativa potenciando el diálogo de saberes. La etnobiología puede contribuir a la resolución de problemas ambientales contemporáneos, pero este potencial no puede realizarse sin una mayor inclusión de los pueblos indígenas y comunidades locales.

L'ethnobiologie étudie les relations que les différentes sociétés établissent avec la nature, en analysant les connaissances, les usages et les perceptions. La Bolivie est un pays présentant une grande diversité biologique et culturelle. Au cours des deux dernières décennies, la Bolivie a initié une politique de défense de l'identité et de la gouvernance autochtones, qui comprend la réévaluation des connaissances écologiques locales. Cette situation offre une excellente opportunité pour le développement et la consolidation de la recherche ethnobiologique dans le pays. Dans cet article, nous documentons les précurseurs biologiques et anthropologiques de l'ethnobiologie bolivienne et nous analysons son évolution à partir de trois études de cas emblématiques : les deux premières montrent la confluence d'un projet biologique et d'un projet anthropologique vers l'ethnobiologie, et la troisième illustre les bénéfices de l'approche participative. La dernière section aborde certains des grands défis de l'ethnobiologie en Bolivie, en se concentrant sur quatre aspects qui permettraient d'établir une base solide pour le développement de la discipline : i) identification des lacunes documentaires et création d'une base de référence; ii) développement méthodologique mettant l'accent sur l'interdisciplinarité, iii) internationalisation; et iv) généralisation de la recherche participative qui permet de renforcer le dialogue des connaissances. L'ethnobiologie peut contribuer à la résolution des problèmes environnementaux contemporains, mais ce potentiel ne peut être réalisé sans une plus grande inclusion des peuples autochtones et des communautés locales. 
Ethnobiology is the study of the relationships that different societies establish with nature, through the anlysis of knowledge, uses and perceptions. Bolivia is a country with great biological and cultural diversity. In the last two decades, Bolivia has initiated a political process in view to defend indigenous identity and governance, which includes the revaluation of local ecological knowledge. This situation provides an excellent opportunity for the development and consolidation of ethnobiological research in the country. In this article, we document the biological and anthropological precursors of Bolivian ethnobiology and we analyze its evolution through three emblematic case studies: the first two show the confluence of a biological and an anthropological project towards ethnobiology, and the third one illustrates the benefits of the participatory approach. The last section addresses some of the major challenges posed by ethnobiology in Bolivia, focusing on four aspects that are necessary to lay a strong foundation for the development of the discipline: i) identification of documentary gaps and creation of a baseline; ii) methodological development with a focus on interdisciplinarity, iii) internationalization; and iv) generalization of participatory research, which helps initiate a dialogue between various types of knowledge. Ethnobiology can contribute to the resolution of contemporary environmental issues, but this potential cannot be realized without a greater inclusion of Indigenous Peoples and local communities.

\section{ÍNDICE}

Mots-clés: anthropologie de l'environnement, connaissances écologiques locales, ethnobotanique, peuples autochtones, Protocole de Nagoya

Keywords: environmental anthropology, ethnobotany, Indigenous Peoples, local ecological knowledge, Nagoya protocol

Palabras claves: antropología ambiental, conocimiento ecológico local, etnobotánica, protocolo de Nagoya, pueblos indígenas

\section{AUTORES}

\section{VICTORIA REYES-GARCÍA}

Institució Catalana de Recerca i Estudis Avançats (ICREA), Barcelona, Spain. Institut de Ciència i Tecnologia Ambientals, Universitat Autònoma de Barcelona, 08193 Bellaterra, Barcelona, España - Departament d'Antropologia Social i Cultural, Universitat Autònoma de Barcelona, 08193 Bellaterra, Barcelona, España. Victoria.reyes@uab.cat

\section{MÓNICA MORAES R.}

Instituto de Ecología, Universidad Mayor de San Andrés, c/ 27 y Av. Andrés Bello s/n, La Paz, Bolivia monicamoraes45@gmail.com - Herbario Nacional de Bolivia, Universidad Mayor de San Andrés, La Paz, Bolivia nyaroslava@yahoo.es

\section{ÁLVARO FERNÁNDEZ-LLAMAZARES}

Helsinki Institute of Sustainability Science (HELSUS), Faculty of Biological and Environmental Sciences, University of Helsinki, FI-00014, Helsinki, Finlandia. alvaro.fernandez-

llamazares@helsinki.fi 


\section{NAREL Y. PANIAGUA-ZAMBRANA}

Herbario Nacional de Bolivia, Universidad Mayor de San Andrés, La Paz, Bolivia nyaroslava@yahoo.es 